\title{
Recognition of durations embedded in temporal patterns
}

\author{
DIANA DEUTSCH \\ University of California, San Diego, La Jolla, California
}

\begin{abstract}
Subjects were presented with a pair of blips which defined a standard duration, followed by another pair of blips, which defined a comparison duration, and they judged whether the comparison was identical to the standard, longer, or shorter. In one set of conditions, a series of extra blips was interpolated during the interstimulus interval, so as to produce interpolated durations in the same range as the standard. Comparison judgments here reflected distortion of memory for the standard in the direction of the interpolated duration. In another set of conditions, the interpolated blips defined durations in the range of half the standard. Comparison judgments here reflected distortion of memory for the standard in the direction of twice the interpolated duration. These results are in accordance with the hypothesis that temporal patterns are distorted by the listener so that they appear closer to those with simple metrical descriptions, and that such distortions occur through a process of memory interference between durations which are embedded in the patterns.
\end{abstract}

Interest has developed recently in the question of how temporal patterns are processed (see, e.g., Fraisse, 1982; Garner, 1974; Jones, 1981; Longuet-Higgins, 1976; Longuet-Higgins \& Lee, 1984; Martin, 1972; Michon, 1974; Monahan \& Carterette, 1985; Povel, 1981, 1984; Povel \& Essens, 1985; Simon \& Sumner, 1968; Steedman, 1977; Sternberg, Knoll, \& Zukofsky, 1982). Much theoretical work on this issue has been influenced by the structure of time in tonal music (Cooper \& Meyer, 1960; Lerdahl \& Jackendoff, 1983; Westergaard, 1975; Yeston, 1976). This is best conceived of in terms of equally spaced reference points, or beats. The time span between two primary beats is called a measure. The measure is in turn divided into two or more equal time spans, which are bordered by secondary beats. The meter is defined by the number of secondary beats dividing the measure. Thus, for example, one secondary beat dividing the measure into equal parts produces duple meter, and two secondary beats produces triple meter. These smaller time spans can themselves be divided into equal parts, and so on. The symbols for notes and rests in tonal music reflect this hierarchical organization. Thus, $\bullet$ denotes a whole note, $d$ a half note, $d$ a quarter note, $d$ an eighth note, $\delta_{\text {a sixteenth }}$ note, and so on.

Rhythmic patterns are thus characterized in terms of metrical hierarchies, which consist of successive divisions of time spans into units of equal length. For example, the sequence of durations (in msec) 1,000-500-500-1,000 forms a plausible and simple rhythmic pattern, and can

This study was supported by USPHS Grant MH-21001 and was reported at the 25th Annual Meeting of the Psychonomic Society in Austin, Texas, 1983. Requests for reprints should be addressed to Diana Deutsch, Department of Psychology, University of California, San Diego, La Jolla, CA 92093. be represented simply in musical notation, as shown in Figure 1A. In contrast, the sequence of durations (in msec) 1,000-700-700-1,000 does not form a plausible rhythmic pattern, and can be represented only in a cumbersome fashion, for example, in the approximate notation shown in Figure 1B. Furthermore, assuming that equal divisions of time spans are involved, the sequence in Figure 1A can be represented in terms of a simple tree diagram, as shown; however, the sequence in Figure 1B cannot be accurately represented in this fashion.

Given that rhythmic patterns in tonal music are organized hierarchically, and given that those which can be simply described in musical notation appear to be readily processed, it is reasonable to suppose that hierarchical subdivisions of time spans are involved in their internal representation also. This supposition gives rise to two separable, though related, issues. The first concerns how the listener assigns rhythmic interpretations to idealized temporal patterns, such as those represented in a musical score or generated by computer. ${ }^{1}$ This issue has been investigated by Simon and Sumner (1968), Martin (1972), Longuet-Higgins (1976), Longuet-Higgins and Lee (1984), Povel and Essens (1985), and others. The second issue concerns the relationship between perception of idealized temporal patterns and perception of those that occur in our natural environment. Do we perceive both types of pattern with the same accuracy, or do we distort naturally occurring patterns in the direction of idealized ones? If the latter is the case, how, specifically, do these distortions occur?

The present paper addresses the second issue. It was hypothesized on general grounds that we do indeed distort temporal patterns so that they appear closer to idealized ones. It was further hypothesized that such distortions result from an ongoing "updating" of earlier 
A

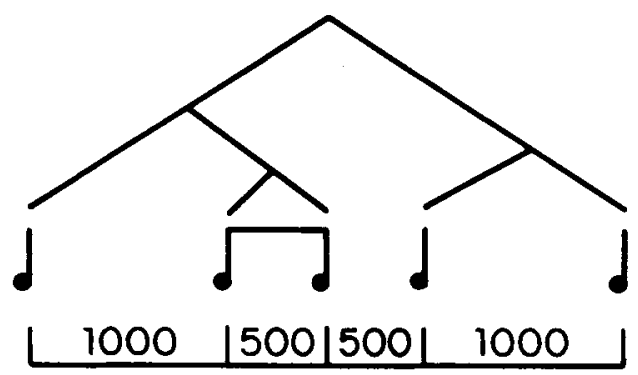

B

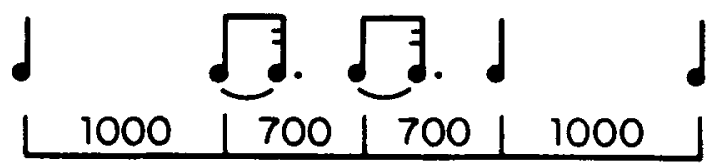

Figure 1. Two temporal patterns: one with a simple metrical structure $(A)$, and one without a clear structure (B).

durations in a pattern in accordance with later durations, so as to enable an encoding in terms of a simple metrical hierarchy. Such a process of memory interference should then be reflected in comparison judgments between durations that are contained in the pattern.

In order to test the above hypotheses, the following paradigm was employed. A pair of blips was presented which defined a standard duration, followed by another pair of blips, which defined a comparison duration. Subjects judged whether the comparison duration was identical to the standard, longer, or shorter.

In one set of conditions, a series of extra blips was interpolated during the interstimulus interval (ISI); these blips defined durations that were in the same range as the standard. It was hypothesized that comparison judgments would reflect distortion of memory for the standard duration in the direction of the interpolated duration. Thus, when the interpolated duration was longer than the standard, there should be a tendency to judge the comparison duration as shorter. Furthermore, when the interpolated duration was shorter than the standard, there should be a tendency to judge the comparison duration as longer.

In another set of conditions, a series of blips was interpolated which defined durations in the range of half the standard. On the assumption that memory distortions would occur in accordance with a metrical hierarchy, it was hypothesized that comparison judgments would here reflect distortion of memory for the standard in the direction of twice the interpolated duration. Thus, when the interpolated duration was longer than half the standard, there should be a tendency to judge the comparison duration as shorter. Furthermore, when the interpolated duration was shorter than half the standard, there should be a tendency to judge the comparison duration as longer. The possibility was also considered that memory distortions would occur based on the direct relationship between the standard and interpolated durations. If this were so, then when the interpolated duration was exactly half the standard, there should be a tendency to judge the comparison as longer.

\section{METHOD}

\section{Procedure}

On each trial, subjects were presented with a pair of blips which marked a standard duration, and then with another pair of blips which marked a comparison duration. The subjects judged on forced choice whether the comparison duration was the same as the standard, longer, or shorter. In two of the three conditions, a series of extra blips was interpolated during the ISI, and the subjects were instructed to ignore the interpolated blips.

\section{Conditions}

The experiment consisted of three conditions, and these were given in separate sessions, the order of presentation being counterbalanced across subjects. The patterns employed in the three conditions are represented schematically in Figure 2 . In Condition 1, two blips were interpolated during the ISI, dividing it into three equal parts and producing interpolated durations in the same range as the standard. In Condition 2, no blips were interpolated during the ISI. In Condition 3, five blips were interpolated, dividing the ISI into six equal parts and producing interpolated durations in the range of half the standard. In all conditions, whenever the standard and comparison durations differed, the two stood in a ratio of 5:4.

Condition 1 consisted of nine subconditions, and these are illustrated in Figure 3. In three of these, the standard and comparison durations were identical. In the first $[C=S(I=S)]$, the interpolated duration was also identical to the standard. In the second $[C=S(I>S)]$, the interpolated duration was longer than the standard. In the third $[C=S(I<S)]$, the interpolated duration was shorter than the standard. Whenever the interpolated duration differed from the standard, the two stood in a ratio of $4: 3$.

In the second three subconditions, the comparison duration was longer than the standard. In the first $[C>S(I=S)]$, the interpolated duration was identical to the standard. In the second $[\mathrm{C}>\mathrm{S}$ $(\mathrm{I}>\mathrm{S})$ ], the interpolated duration was longer than the standard. In the third $[C>S(I<S)]$, the interpolated duration was shorter than the standard. Again, when the interpolated duration differed from the standard, the two stood in a ratio of $4: 3$. Thus, when the interpolated duration was longer than the standard, it was also very similar to the comparison duration.

In the final three subconditions, the comparison duration was shorter than the standard. In the first $[C<S(I=S)]$, the interpolated duration was identical to the standard. In the second $[C<S$ (I $>$ S)], the interpolated duration was longer than the standard.
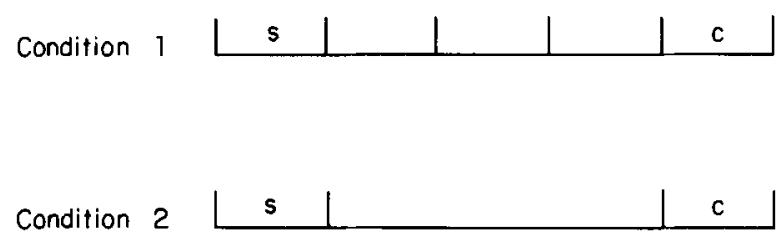

Condition 3

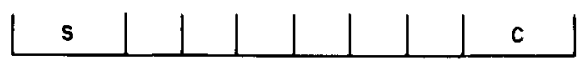

Figure 2. Temporal patterns employed in the different conditions of the experiment. For each of these patterns, the duration of the interstimulus interval was varied, as was the comparison duration. $\mathrm{S}=$ standard duration. $\mathrm{C}=$ comparison duration. 


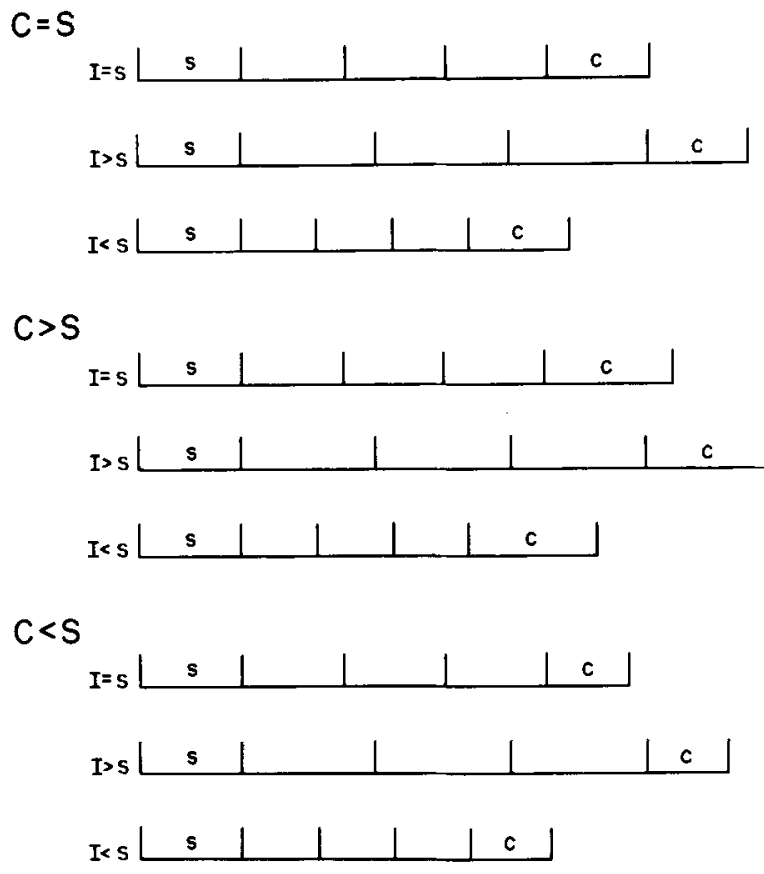

Figure 3. Patterns used in the different subconditions of Condition 1 . See text for details. $S=$ standard duration. $C=$ comparison duration. $I$ = interpolated duration.

In the third $[\mathrm{C}<\mathrm{S}(\mathrm{I}<\mathrm{S})]$, the interpolated duration was shorter than the standard. Again, when the interpolated duration differed from the standard, the two stood in a ratio of $4: 3$. Thus, when the interpolated duration was shorter than the standard, it was also very similar to the comparison duration.

Condition 2 also consisted of nine subconditions. The stimulus parameters in these subconditions were identical to those in Condition 1, except that no blips were interpolated during the ISI. For example, in subcondition $\mathrm{C}=\mathrm{S}(\mathrm{I}=3 \mathrm{~S})$, the comparison duration was identical to the standard, and the interpolated duration was three times as long. The remaining subconditions were analogously defined.

Condition 3 again consisted of nine subconditions. The stimulus parameters were identical to those of Condition 1, except that now five blips were interpolated during the ISI, so as to define durations which were half as long as those in Condition 1 . Thus, for example, in subcondition $C=S(I=1 / 2 S)$, the comparison duration was identical to the standard, and the interpolated duration was half as long. In subcondition $C=S(I>1 / 2 S)$, the comparison duration was identical to the standard, and the interpolated duration was $4 / 3$ of half the standard (i.e., $2 / 3$ of the standard). In subcondition $\mathbf{C}=\mathbf{S}(\mathbf{I}<1 / 2 S)$, the comparison duration was identical to the standard, and the interpolated duration was $3 / 4$ of half the standard, (i.e., $3 \%$ of the standard). The remaining subconditions were analogously defined.

\section{Stimulus Parameters}

All blips were $1000-\mathrm{Hz}$ sine-wave tones, at equal amplitude, and were 50 msec in duration. Durations were defined by the time elapsing between blip onsets. For each subcondition, the standard duration was $1,000 \mathrm{msec}, 1,200 \mathrm{msec}$ or $1,500 \mathrm{msec}$; each of these values occurred equally often. ${ }^{2}$ The comparison and interpolated durations were then as specified for the subcondition. In each session, all 27 patterns were presented twice, making 54 trials in all. These were presented in blocks of nine, with 10 -sec pauses between trials within a block, and 2-min pauses between blocks. Sequences within each session were presented in random order.

\section{Apparatus}

Tones were generated by a Wavetek function generator (Model No. 155) controlled by a PDP $11 / 23$ computer, and were recorded on tape. The tape was played to subjects on a Revox tape recorder, the output of which was passed through a Crown amplifier and presented to subjects through a loudspeaker. At the beginning of each session, the task was explained to the subjects, and they were given three practice trials.

\section{Subjects}

Eighteen undergraduates at the University of California, San Diego, served as subjects in the experiment. They had all had at least two years of musical training; there was otherwise no selection procedure. The subjects were paid for their services.

\section{RESULTS}

Figures 4, 5, and 6 display performance levels in the different conditions of the experiment, plotted separately for subconditions in which the comparison duration was identical to the standard $(C=S)$, it was longer $(C>S)$, and it was shorter $(C<S)$. It can be seen that when blips were interpolated which defined durations either in the same range as the standard (Condition 1) or in the range of half the standard (Condition 3), performance levels varied substantially depending on the relationship of the interpolated duration to the standard. However, little variation is apparent when ISIs contained no interpolated blips (Condition 2).

Three-way ANOVAs were performed to compare performance levels in the $\mathrm{C}=\mathrm{S}, \mathrm{C}>\mathrm{S}$, and $\mathrm{C}<\mathrm{S}$ subconditions separately, with number of interpolated blips and ISI duration as fixed factors and subjects as a random factor. For $\mathrm{C}=\mathrm{S}$, the effect of number of blips was highly significant $[F(2,34)=27.34, p<.001]$, as was the effect of ISI duration $[F(2,34)=28.01, p<.001]$ and the interaction $[F(4,68)=10.90, p<.001]$. Similarly, for $\mathrm{C}>\mathrm{S}$, the effect of number of blips $[F(2,34)$ $=5.10, p=.01]$, the effect of ISI duration $[F(2,34)=$ $46.55, p<.001]$, and the interactions $[F(4,68)=14.35$, $p<.001$ ] were all highly significant. Again, for $\mathrm{C}<\mathrm{S}$, the effect of number of blips $[F(2,34)=17.30, p<.001]$ the effect of ISI duration $[F(2,34)=40.56, p<.001]$, and the interaction $[F(4,68)=13.19, p<.001]$ were all highly significant.

Two-way ANOVAs, taking Conditions 1, 2, and 3 separately, confirmed that variations in performance level depending on ISI duration occurred only when blips were interpolated. The effect of ISI duration was highly significant in Condition $1[\mathrm{C}=\mathrm{S}, F(2,34)=32.42, p<$ $.001 ; \mathrm{C}>\mathrm{S}, F(2,34)=43.86, p<.001 ; \mathrm{C}<\mathrm{S}$, $F(2,34)=30.57, p<.001]$ and in Condition $3[\mathrm{C}=\mathrm{S}$, $F(2,34)=10.46, p<.001 ; \mathrm{C}>\mathrm{S}, F(2,34)=22.14$, $p<.001 ; \mathrm{C}<\mathrm{S}, F(2,34)=28.27, p<.001]$. However, the effect was nonsignificant in Condition 2 
$\mathrm{C}=\mathrm{S}$
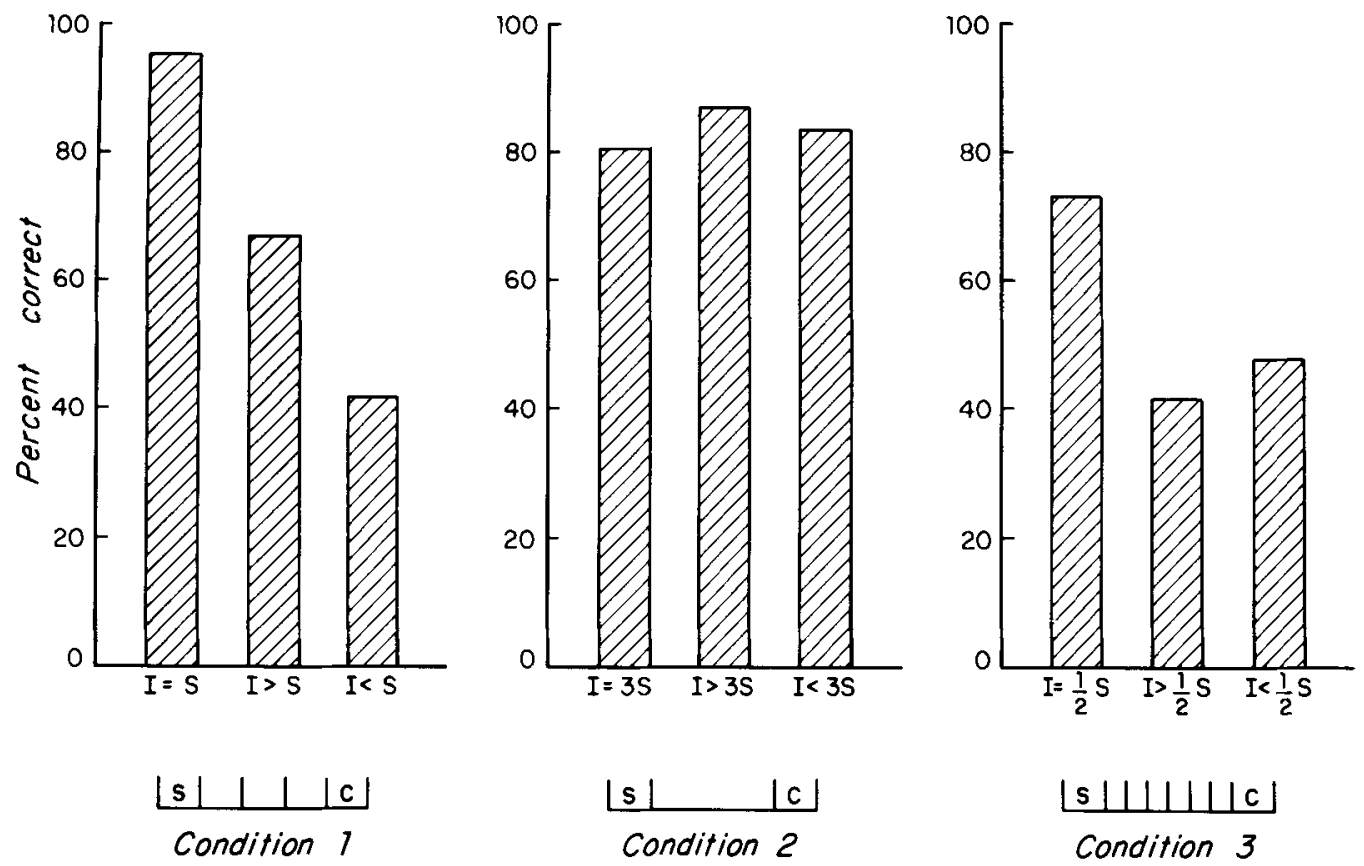

Figure 4. Performance levels in the different conditions of the experiment (taking subconditions in which the standard and comparison durations were identical).

$C>S$
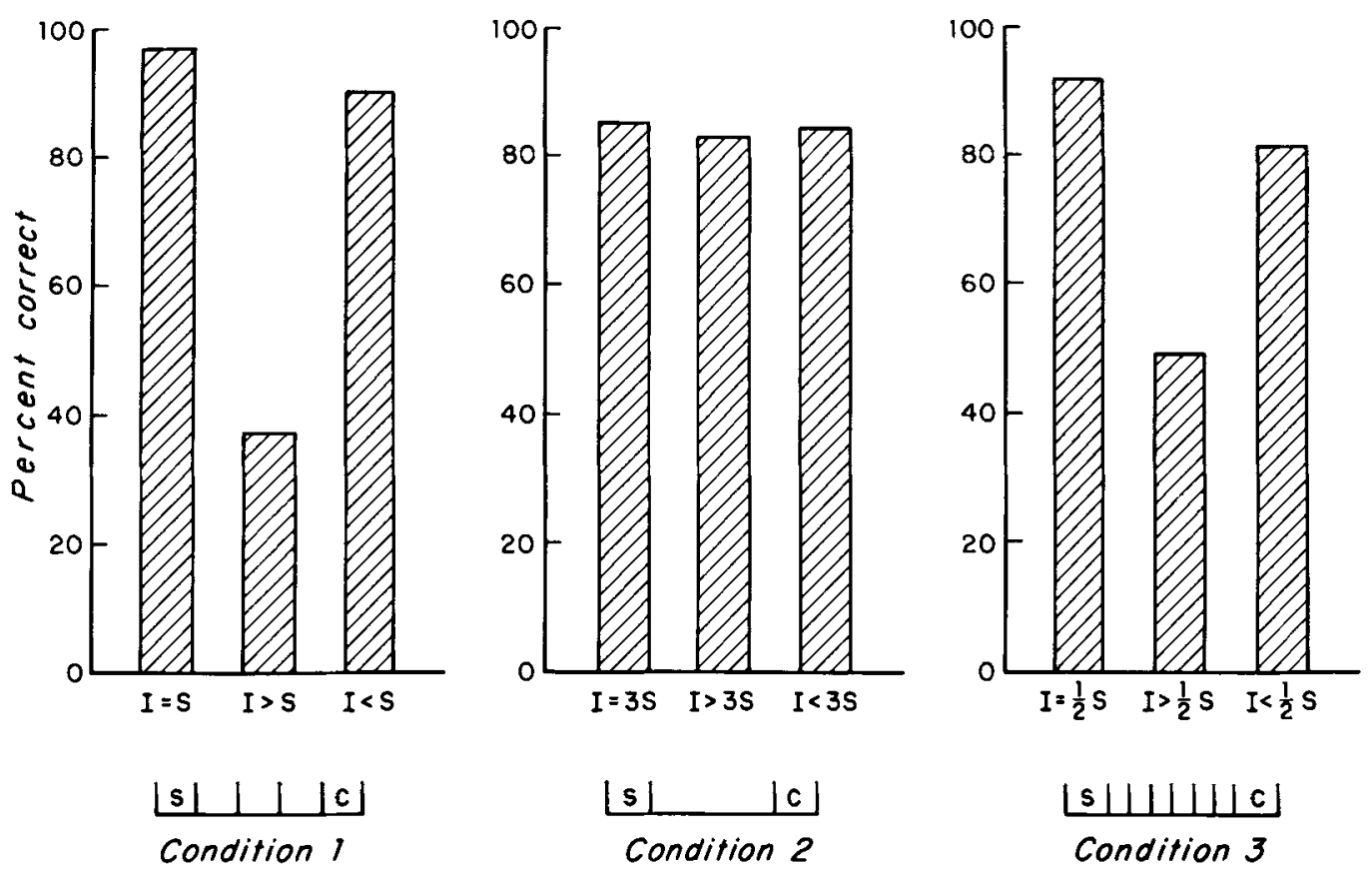

Figure 5. Performance levels in the different conditions of the experiment (taking subconditions in which the comparison duration was longer than the standard). 

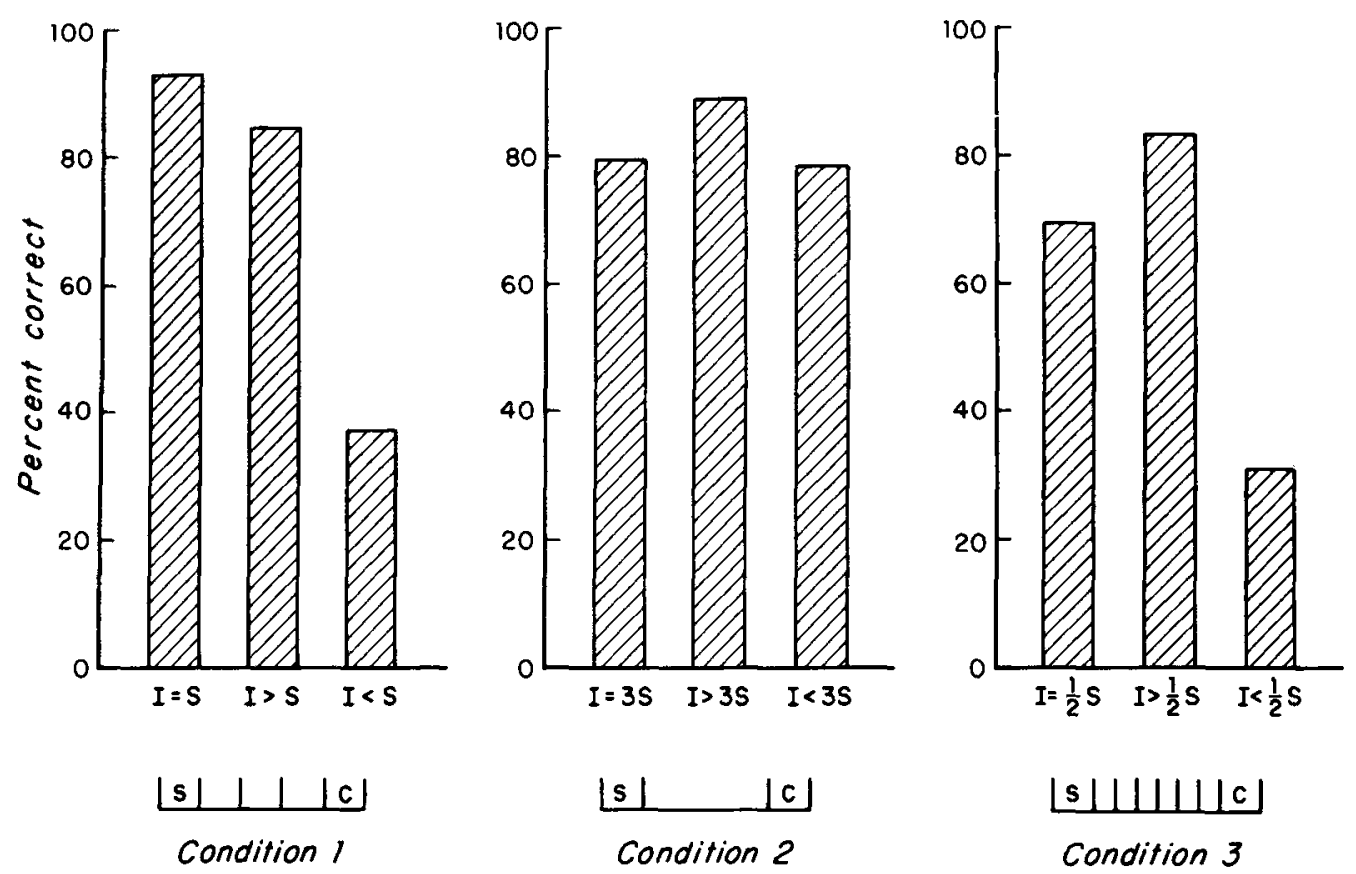

Figure 6. Performance levels in the different conditions of the experiment (taking subconditions in which the comparison duration was shorter than the standard).

$[\mathrm{C}=\mathrm{S}, F<1 ; \mathrm{C}>\mathrm{S}, F<1 ; \mathrm{C}<\mathrm{S}, F(2,34)=$ $3.01, p>.05]$. We turn, therefore, to a detailed analysis of performance in Conditions 1 and 3.

\section{Condition 1}

Performance levels when the standard and comparison durations were identical are displayed in the left-hand portion of Figure 4. It can be seen that when the interpolated duration was also identical to the standard, a very high level of performance was obtained. Performance was considerably poorer when the interpolated duration was longer than the standard, and also when it was shorter.

This leads us to inquire whether the enhanced error rates in the latter two subconditions were due simply to general reduction in accuracy, or rather to distortions of memory for the standard in the direction of the interpolated duration. Table 1 displays the percentages of "longer" and "shorter" judgments in the different subconditions. It can be seen that the enhanced error rates can be accounted for entirely by the hypothesis of memory distortion. In subcondition $\mathrm{C}=\mathrm{S}(\mathrm{I}>\mathrm{S})$, in which the interpolated duration was longer than the standard, the number of "longer" judgments was minimal, as it was in subcondition $C=S(I=S)$, in which the interpolated duration was identical to the standard. In contrast, the number of "shorter" judgments was substantially larger in subcondition $\mathrm{C}=\mathrm{S}(\mathrm{I}>\mathrm{S})$ than in the subcondition $\mathrm{C}=\mathrm{S}$ $(I=S)$. The difference between these values was highly significant $[F(1,17)=30.23, p<.001]$. Similarly, in subcondition $\mathrm{C}=\mathrm{S}(\mathrm{I}<\mathrm{S})$, in which the interpolated duration was shorter than the standard, the number of "shorter" judgments was minimal, as it was in subcon- dition $C=S(I=S)$. However, the number of "longer" judgments was substantially larger in subcondition $C=S$ $(I<S)$ than in subcondition $\mathrm{C}=\mathrm{S}(\mathrm{I}=\mathrm{S})$. This difference was also highly significant $[F(1,17)=73.91, p<$ $.001]$.

Performance levels in the subconditions in which the comparison duration was longer than the standard are displayed in the left-hand portion of Figure 5. It can be seen that when the interpolated duration was identical to the standard, the performance level was very high. This was true also when the interpolated duration was shorter than the standard. However, when it was longer, so that it was similar to the comparison, performance was virtually at chance. As shown in Table 2, the additional errors con-

Table 1

Percentages of "Longer" and "Shorter" Judgments in Conditions 1 and $3, \mathrm{C}=\mathrm{S}$

\begin{tabular}{cccc} 
Condition & Subcondition & Judgment & $\begin{array}{c}\text { Percentage of } \\
\text { Responses }\end{array}$ \\
\hline 1 & $\mathrm{C}=\mathrm{S}(\mathrm{I}=\mathrm{S})$ & "Longer" & 0.0 \\
& & "Shorter" & 4.6 \\
& $\mathrm{C}=\mathrm{S}(\mathrm{I}>\mathrm{S})$ & "Longer" & 1.9 \\
& & "Shorter" & 31.5 \\
& $\mathrm{C}=\mathrm{S}(\mathrm{I}<\mathrm{S})$ & "Longer" & 55.6 \\
& & "Shorter" & 2.8 \\
3 & $\mathrm{C}=\mathrm{S}(\mathrm{I}=1 / 2 \mathrm{~S})$ & "Longer" & 20.3 \\
& & "Shorter" & 6.5 \\
& $\mathrm{C}=\mathrm{S}(\mathrm{I}>1 / 2 \mathrm{~S})$ & "Longer" & 19.4 \\
& & "Shorter" & 37.0 \\
& $\mathrm{C}=\mathrm{S}(<1 / 2 \mathrm{~S})$ & "Longer" & 45.4 \\
& & "Shorter" & 6.5 \\
\hline
\end{tabular}


Table 2

Percentages of "Same" Judgments in Conditions 1 and $3, C \neq S$

\begin{tabular}{clc} 
Condition & Subcondition & $\begin{array}{c}\text { Percentage of } \\
\text { Responses }\end{array}$ \\
\hline 1 & $\mathrm{C}>\mathrm{S}(\mathrm{I}=\mathrm{S})$ & 3.7 \\
& $\mathrm{C}>\mathrm{S}(\mathrm{I}>\mathrm{S})$ & 60.2 \\
& $\mathrm{C}>\mathrm{S}(\mathrm{I}<\mathrm{S})$ & 9.3 \\
& $\mathrm{C}<\mathrm{S}(\mathrm{I}=\mathrm{S})$ & 6.5 \\
& $\mathrm{C}<\mathrm{S}(\mathrm{I}>\mathrm{S})$ & 14.8 \\
& $\mathrm{C}<\mathrm{S}(\mathrm{I}<\mathrm{S})$ & 61.1 \\
3 & $\mathrm{C}>\mathrm{S}(\mathrm{I}=1 / 2 \mathrm{~S})$ & 7.4 \\
& $\mathrm{C}>\mathrm{S}(\mathrm{I}>1 / 2 \mathrm{~S})$ & 53.7 \\
& $\mathrm{C}>\mathrm{S}(\mathrm{I}<1 / 2 \mathrm{~S})$ & 16.7 \\
& $\mathrm{C}<\mathrm{S}(\mathrm{I}=1 / 2 \mathrm{~S})$ & 28.7 \\
& $\mathrm{C}<\mathrm{S}(\mathrm{I}>1 / 2 \mathrm{~S})$ & 10.2 \\
& $\mathrm{C}<\mathrm{S}(\mathrm{I}<1 / 2 \mathrm{~S})$ & 67.6 \\
\hline
\end{tabular}

sisted overwhelmingly of judgments that the standard and comparison durations were identical, and this again indicates that memory for the standard was being distorted in the direction of the interpolated duration. The increase in the number of "same" judgments was highly significant $[C>S(I>S)$ vs. $C>S(I=S), F(1,17)=$ 28.87, $p<.001 ; \mathrm{C}>\mathrm{S}(\mathrm{I}>\mathrm{S})$ vs. $\mathrm{C}>\mathrm{S}(\mathrm{I}<\mathrm{S})$, $F(1,17)=38.12, p<.001]$.

Performance levels in the subconditions in which the comparison duration was shorter than the standard are displayed in the left-hand portion of Figure 6. It can be seen that, again, when the standard and interpolated durations were identical, a very high level of performance was obtained. This was true also when the interpolated duration was longer than the standard. However, when it was shorter, so that it was similar to the comparison, performance was considerably poorer. As shown in Table 2, the additional errors consisted overwhelmingly of judgments that the standard and comparison durations were identical, indicating that memory for the standard was being distorted in the direction of the interpolated duration. The increase in the number of "same" judgments was also highly significant $[\mathrm{C}<\mathrm{S}(\mathrm{I}<\mathrm{S})$ vs. $\mathrm{C}<\mathrm{S}$ (I = S), $F(1,17)=87.41, p<.001 ; \mathrm{C}<\mathrm{S}(\mathrm{I}<\mathrm{S})$ vs. $\mathrm{C}<\mathrm{S}(\mathrm{I}>\mathrm{S}), F(1,17)=22.46, p<.001]$.

\section{Condition 3}

Performance levels when the standard and comparison durations were identical are displayed in the right-hand portion of Figure 4. It can be seen that when the interpolated duration was equal to half the standard, the performance level was substantially higher than when it was longer or shorter than half the standard.

This leads us again to inquire whether the patterns of error were due to the simple differences in performance accuracy or to systematic distortions of memory for the standard. As discussed in the introduction, such distortions could in turn come from two different sources. First, they could be based directly on the relationship between the interpolated duration and the standard. Second, assuming that a metrical hierarchy was involved, they could be based on the relationship of the interpolated duration to half the standard. The first source of distortion should produce a tendency to judge the comparison duration as longer than the standard in all three subconditions. The second source should produce no effect in subcondition $C=S(I=1 / 2 S)$, should produce a tendency to judge the comparison as shorter in subcondition $C=S(I>1 / 2 S)$, and should produce a tendency to judge the comparison as longer in subcondition $C=S(I<1 / 2 S)$.

Table 1 displays the percentages of "longer" and "shorter" judgments in the different subconditions. It can be seen that both sources of distortion were operating here. The number of "longer" judgments was significantly larger in subcondition $C=S(I=1 / 2 S)$ than in subcondition $C$ $=\mathrm{S}(\mathrm{I}=\mathrm{S})[F(1,17)=34.86, p<.001]$. However, the number of "shorter" judgments was minimal in both these subconditions. This pattern is in accordance with the presence of memory distortion based directly on the relationship between the standard and interpolated durations. The number of "longer" judgments was also significantly larger in subcondition $\mathrm{C}=\mathrm{S}(\mathrm{I}>1 / 2 S)$ than in subcondition $\mathrm{C}=\mathrm{S}(\mathrm{I}>\mathrm{S})[F(1,17)=14.61, p<.002]$, again as expected on the basis of this hypothesis.

The hypothesis of distortion based on a metrical hierarchy leads to the further prediction of a larger number of "shorter" judgments in subcondition $C=S(I>1 / 2 S)$ compared with subcondition $C=S(I=1 / 2 S)$. Indeed, a highly significant effect in this direction was found $[F(1,17)=19.59, p<.001]$. Both the direct hypothesis and the metrical hypothesis predict a larger number of "longer" judgments in subcondition $C=S(I<1 / 2 S)$ than in subcondition $C=S(I=1 / 2 S)$. A highly significant effect in this direction was also found $[F(1,17)=$ $17.00, p<.001]$.

Performance levels in subconditions in which the comparison duration was longer than the standard are shown in the right-hand portion of Figure 5. It can be seen that when the interpolated duration was identical to half the standard, a high level of performance was achieved; this was also true when the interpolated duration was shorter than half the standard. However, when it was longer, so that it was closely similar to twice the comparison, performance was substantially poorer. As shown in Table 2, the additional errors consisted overwhelmingly of judgments that the comparison duration was identical to the standard, again indicating that memory for the standard was being distorted in the direction of twice the interpolated duration. This effect is as predicted by the metrical hypothesis. The increase in the number of "same"' judgments was here highly significant $[C>S(I>1 / 2 S)$ vs. $\mathrm{C}>\mathrm{S}(\mathrm{I}=1 / 2 \mathrm{~S}), F(1,17)=36.43, p<.001 ; \mathrm{C}>\mathrm{S}$ (I $>1 / 2 S)$ vs. $C>S(I<1 / 2 S), F(1,17)=18.90, p<$ $.001]$.

Performance levels in the subconditions in which the interpolated duration was shorter than half the standard are shown in the right-hand portion of Figure 6. It can be seen that the performance level was very high when 
the interpolated duration was longer than half the standard and when it was equal to half the standard. However, when it was shorter, so that it was closely similar to half the comparison, performance fell below chance. As displayed in Table 2, the additional errors consisted largely of judgments that the comparison was identical to the standard, again indicating that memory for the standard was being distorted in the direction of twice the interpolated duration. This increase in the number of "same" judgments was highly significant $[C<S(I<1 / 2 S)$ vs. $\mathrm{C}<\mathrm{S}(\mathrm{I}=1 / 2 \mathrm{~S}), F(1,17)=20.73, p<.001 ; \mathrm{C}<\mathrm{S}$ $(\mathrm{I}<1 / 2 \mathrm{~S})$ vs. $\mathrm{C}<\mathrm{S}(\mathrm{I}>1 / 2 \mathrm{~S}), F(1,17)=52.00, p<$ $.001]$.

\section{DISCUSSION}

The major findings of the experiment confirm the hypothesis that temporal patterns are distorted by the listener so that they appear closer to idealized ones, and that such distortions occur through a process of memory interference between the durations embedded in the patterns. $^{3}$ Phenomena such as those described here place clear constraints on the types of pattern which can be accurately perceived by the listener. We note that the framework for producing rhythmic patterns in tonal music is such as to accommodate these constraints, and so we may conjecture that such a framework has evolved to make optimal use of our temporal processing mechanisms.

The findings also show, as an additional and subsidiary effect, that memory distortions occur which are based on the direct relationships between the interacting durations. Thus, it appears that temporal pattern representations resulting from the two combined sources of distortion are not in fact strictly idealized, but rather are slight distortions of idealized patterns. This finding would not be expected from music-theory considerations, and has implications for the interpretation of certain inaccuracies in performance. Strictly timed, or "deadpan," musical performances, such as those usually generated by computer, tend to sound incorrect in some way. We may hypothesize from the present findings that certain mistimings in live performances occur as compensations for perceptual and mnemonic biases of the sort described here. For example, we might expect that the sequence shown in Figure 7 would be played with the marked note slightly shorter than indicated, and that such a rendition would sound more accurate than one which was exactly timed.

This leads us to the general issue of toleration of deviations from strict timing in musical performance. Good performances often differ considerably from each other, and yet the listener accepts each different version as correct. However, there are other performances in which deviations from accuracy are no greater, but appear as errors instead. We may hypothesize that such differences in judgment are based, at least in part, on phenomena such as those described here. For example, when a duration is repeated exactly following the interpolation of slightly longer durations, it will appear the second time to be

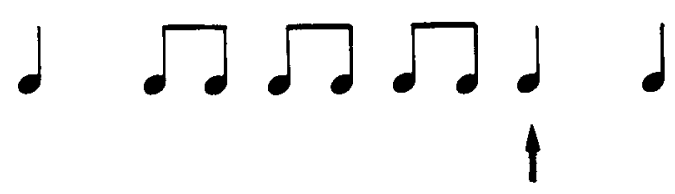

Figure 7

shorter. Therefore, if this duration is lengthened slightly on repetition, it will appear to have been accurately produced. However, if instead it is shortened on repetition, it will appear the second time to be clearly shorter. Such effects can be put to various uses. For example, one can produce a gradual shift in tempo so that the listener is unable to pinpoint any single duration as different from previous ones, but simply becomes aware of a global change.

The present findings may be related to those concerning accuracy of production of temporal patterns. In general, it has been found that patterns which can be described in terms of a simple metrical hierarchy can be accurately produced, and that those which cannot be so described are generated more poorly. Fraisse (1982) reported classical work showing that subjects have considerable difficulty in generating irregular sequences, tending instead to produce sequences with interresponse intervals which are roughly identical or which stand in a ratio of 2:1. More recently, Povel (1981) required subjects to imitate various temporal patterns, and found that patterns which had a simple metrical description were well imitated, and those which did not were inaccurately imitated. In another study, Deutsch (1983) presented subjects with two simultaneous pulse trains, and required them to tap along with both in parallel. When the intervals associated with the two trains stood in an integral multiple relationship, high levels of accuracy were achieved. However, when the intervals did not stand in this relationship, performance was considerably poorer. Furthermore, accuracy in generating patterns of the latter type was directly related to the simplicity of their description in terms of a metrical hierarchy (see also Kelso, Southard, \& Goodman, 1979; Klapp, 1979; Peters, 1977; Summers, 1975).

Studies of production alone, however, leave open the question of the origin of these constraints in the generation of temporal patterns. They could, for example, stem from some property of the motor system. However, we may argue that inasmuch as analogous constraints have been found in the present experiment to exist at the levels of perception and memory, the two sets of constraints may have a common origin. (See Sternberg, Knoll, \& Zukofsky, 1982, for an extended discussion of the relationship between perception and performance in temporal processing.)

Finally, analogies may be drawn between the present findings and earlier findings concerning short-term memory for pitch (Deutsch, 1972a, 1972b, 1975). When comparison judgments are made between two tones which are separated by a retention interval, the interpolation of 
a series of extra tones during this interval can produce substantial memory interference. This is true even when the subject has been instructed to ignore the interpolated tones. The degree of such interference depends critically on the pitch relationships between the standard and interpolated tones, and also on the relationships between the interpolated and comparison tones. For example, when the standard and comparison tones differ, and a tone is interpolated which is identical or closely similar to the comparison, this produces a tendency to judge the comparison as identical to the standard (Deutsch, 1972a, 1975). This is analogous to the misrecognition effect found in the present experiment for the case of duration. In the case of pitch, it was further shown (Deutsch, 1972b, 1975) that the degree of interference varies precisely and systematically as a function of similarity between the standard and interpolated tones. Whether a similar function can be demonstrated in the case of short-term memory for duration remains to be investigated.

\section{REFERENCES}

COOPER, G. W., \& MEYER, L. B. (1960). The rhythmic structure of music. Chicago: University of Chicago Press.

DeUTSCH, D. (1972a). Effect of repetition of standard and comparison tones on recognition memory for pitch. Journal of Experimental Psychology, 93, 156-162.

DEUTSCH, D. (1972b). Mapping of interaction in the pitch memory store. Science, 175, 1020-1022.

DEUTSCH, D. (1975). The organization of short-term memory for a single acoustic attribute. In D. Deutsch \& J. A. Deutsch (Eds.), Short term memory (pp. 107-151). New York: Academic Press.

DEuTsCH, D. (1983). The generation of two isochronous sequences in parallel. Perception \& Psychophysics, 34, 331-337.

FraIsse, P. (1982). Rhythm and tempo. In D. Deutsch (Ed.), The psychology of music. New York: Academic Press.

GARNER, W. R. (1974). The processing of information and structure. Hillsdale, NJ: Erlbaum.

JONES, M. R. (1981). Only time can tell: On the topology of mental space and time. Critical Inquiry, 7, 557-576.

Kelso, J. A. S., Southhard, D. L., \& Goodman, D. (1979). On the coordination of two-handed movements. Journal of Experimental Psychology: Human Perception \& Performance, 5, 229-238.

KLAPP, S. T. (1979). Doing two things at once: The role of temporal compatibility. Memory \& Cognition, 7, 375-381.

LERDAHL, F., \& JACKENDOFF, R. A. (1983). A generative theory of tonal music. Cambridge, MA: MIT Press.

LoNGUET-Higgins, H. C. (1976). The perception of melodies. Nature (London), 263, 646-653.
Longuet-Higgins, H. C., \& LeE, C. S. (1984). The rhythmic interpretation of monophonic music. Music Perception, 1, 424-441.

MARTIN, J. G. (1972). Rhythmic (hierarchical) versus serial structure in speech and other behaviour. Psychological Review, 79, 487-509.

MichON, J. A. (1974). Programs and "programs" for sequential patterns in motor behavior. Brain Research, 71, 413-424.

Monahan, C. B., \& Carterette, E. C. (1985). Pitch and duration as determinants of musical space. Music Perception, 3, 1-33.

Peters, M. (1977). Simultaneous performance of two motor activities: The factor of timing. Neuropsychologia, 15, 461-465.

Povel, D.-J. (1981). Internal representation of simple temporal patterns. Journal of Experimental Psychology: Human Perception \& Performance, 7, 3-18.

PoveL, D.-J. (1984). A theoretical framework for rhythm perception. Psychological Research, 45, 315-337.

Povel, D.-J., \& Essens, P. (1985). Perception of temporal patterns. Music Perception, 2, 411-440.

Simon, H. A., \& Sumner, R. K. (1968). Pattern in music. In B. Kleinmuntz (Ed.), Formal representation of human judgment. New York: Wiley.

Steedman, M. J. (1977). The perception of musical thythm and metre. Perception, 6, 555-569.

SternberG, S., Knoll, R. L., \& Zukofsky, P. (1982). Timing by skilled musicians. In D. Deutsch (Ed.), The psychology of music. New York: Academic Press.

Summers, J. J. (1975). The role of timing in motor program representation. Journal of Motor Behavior, 7, 229-241.

WestergaARD, P. (1975). An introduction to tonal theory. New York: Norton.

YESTON, M. (1976). The stratification of musical rhythm. New Haven: CT: Yale University Press.

\section{NOTES}

1. Throughout this paper, the term idealized temporal pattern is used to refer to patterns which allow simple descriptions in terms of divisions of time spans into units of equal length.

2. It should be noted that the values of the standard, comparison, and interpolated durations were all in the range within which the organization of durations into rhythmic patterns is assumed to occur (Fraisse, 1982; Lerdahl \& Jackendoff, 1983).

3. It might be suggested that the present results could be explained by the subjects' mistakenly judging the comparison duration relative to the interpolated duration rather than to the standard duration. However, this hypothesis cannot account for the substantial superiority in performance in condition $I=1 / 2 S$ compared with conditions $I>1 / 2 S$ and $I<1 / 2 S$, because in all these conditions the interpolated duration was considerably shorter than the standard.

(Manuscript received February 4, 1985; revision accepted for publication February 20, 1986.) 